\title{
HAMMERSTEIN, Notker, WALTHER, Gerrit, Späthumanismus. Studien über das Ende einer kulturhistorischen Epoche
}

Jean-Luc Le Cam

\section{(2) OpenEdition}

Journals

Édition électronique

URL : http://journals.openedition.org/ifha/1115

DOI : $10.4000 /$ ifha. 1115

ISSN : 2198-8943

Éditeur

IFRA - Institut franco-allemand (sciences historiques et sociales)

Référence électronique

Jean-Luc Le Cam, «HAMMERSTEIN, Notker, WALTHER, Gerrit, Späthumanismus. Studien über das Ende einer kulturhistorischen Epoche », Revue de l'IFHA [En ligne], Date de recension, mis en ligne le 01 janvier 2003, consulté le 22 septembre 2020. URL : http://journals.openedition.org/ifha/1115 ; DOI : https:// doi.org/10.4000/ifha.1115

Ce document a été généré automatiquement le 22 septembre 2020.

(CIFHA 


\section{HAMMERSTEIN, Notker, WALTHER, Gerrit, Späthumanismus. Studien über das Ende einer kulturhistorischen Epoche}

Jean-Luc Le Cam

1 L'humanisme est un des plus importants et anciens concepts de l'histoire culturelle de la période moderne. Si l'on s'accorde pour situer l'origine de ce phénomène en Italie peu après 1300 et à suivre son extension dans le reste de l'Europe au cours des deux siècles suivants, on a du mal à lui fixer une borne chronologique en aval. Pendant longtemps, on considéra que le mouvement s'épuisait, ou s'affadissait dans la routine scolaire, au milieu du XVIe s. L'historiographie traditionnelle du protestantisme avait tendance à considérer que l'humanisme s'achevait quand il avait rempli sa fonction historique de creuset de la Réforme. La focalisation sur la confessionnalisation ces deux dernières décennies a contribué indirectement à prolonger cette vision. Pour autant, il a toujours existé quelques voix pour dire la force de la pensée humaniste encore au tournant des années 1600 et à la veille de la guerre de Trente Ans. D'où l'invention du terme Späthumanismus (humanisme tardif) pour désigner cet été de la Saint-Martin. Il a d'abord été forgé par les germanistes, tel Erich Trunz, qui avait développé l'idée d'un humanisme tardif comme culture des élites (Standeskultur) vers 1600. Il fut ensuite repris par certains historiens : Gerhard Oestreich et Wilhelm Kühlemann ont su par exemple montrer la part de l'humanisme dans la rénovation de la pensée politique au début du XVIIe s. Mais le terme ne s'est guère acclimaté en-dehors de la sphère germanique.

2 C'est pour explorer ce concept particulier et plus généralement la question de la fin de l'humanisme et sa délimitation d'avec le début du baroque que fut réuni en septembre 1998 ce colloque à l'université de Tübingen, conçu comme la première phase d'un programme de recherche financé par la fondation Gerda Henkel. Ce livre reprend les 15 contributions d'historiens, de littéraires et d'historiens de l'art, sans prétention à 
l'exhaustivité car il reste encore des pans de la question dans l'ombre faute de recherches appropriées.

N.H., éminent spécialiste d'histoire des universités et des sciences, se charge d'ouvrir l'ouvrage par un point historiographique sur le concept d'humanisme tardif et les questions qu'il pose. M. LANZINNER se voit confier la tâche ingrate d'esquisser les grandes tendances de l'histoire politique de l'Empire de 1555 à 1618 , ce qu'il fait très bien en mettant six points en exergue. Le cadre étant ainsi posé, N. MOUT peut s'attaquer à la recherche des tendances humanistes dans les activités de la cour impériale, de Maximilien II à Rodolphe II, tandis que D. MERTENS compare sous le même point de vue les cours princières d'Heidelberg et de Stuttgart, l'une calviniste, l'autre luthérienne. A. SCHMID choisit plutôt de traiter de l'humanisme à la cour de Bavière sous l'angle de l'historiographie dynastique en cherchant à démêler les entreprises qui relèvent de l'humanisme tardif de celles qui annoncent le baroque. G.W., l'un des deux co-éditeurs de l'ouvrage, traite un des aspects majeurs de la problématique qui est le rapport entre humanisme et confession religieuse. Ses conclusions sont nuancées : la dynamique du confessionnel n'a pas asservi ou écrasé celle de l'humanisme, les deux mouvements ont su coexister et se sont modérés et relativisés l'un l'autre. Il est regrettable cependant que ne soit pas du tout évoquée l'opposition entre théologiens orthodoxes et " philosophes " humanistes héritiers de Melanchthon (querelle d'Hofmann à Helmstedt ou guerre théologique entre universités). Elle retoucherait sensiblement ce tableau. F. RÄDLE présente l'humanisme de la Contre-Réforme à travers le théâtre scolaire des jésuites, dont les thèmes exaltent la culture humaniste comme nouvelle noblesse au service de la religion. Une escapade hors de l'Empire vers les îles britanniques nous est offerte par A. ASSMANN, qui souligne le fond typiquement humaniste de deux penseurs opposés du XVIIe s. anglais : le puritain John Milton et le sceptique Thomas Browne. La coloration néo-stoïcienne de cet humanisme tardif, déjà signalée par Gerhard Oestreich, est explorée dans les communications d'U. MUHLACK sur le " tacitisme " et de C. WIEDEMANN sur Juste Lipse, à travers une étude stylistique de sa Politique, tissée de citations antiques comme un cento (collage de citations). Par ces deux contributions, on touche à ce qui semble une particularité de cette dernière phase de l'humanisme : son intérêt et sa fécondité pour la science politique naissante.

4 A. STANNEK fait ensuite le point sur le voyage de cavalier, comme forme adaptée à la noblesse de peregrinatio academica, confortant sa distinction sociale. I. MACLEAN s'intéresse à la présence de l'humanisme dans les disciplines juridiques et médicales. $\mathrm{K}$. REICHERT présente à travers le cas de John Dee la frontière ténue qui sépare à cette époque l'activité scientifique de la magie. Les deux dernières communications recherchent ce que pourrait signifier pour l'histoire de l'art l'application du concept de Späthumanismus à cette période qu'elle désigne comme maniérisme. A. TÖNNESMANN (co-auteur avec Daniel Arasse du volume de la collection "Univers des Formes " consacré au maniérisme) montre à travers l'exemple de la statuaire politique de la Piazza della Signoria à Florence tout au long du XVIe s. l'autonomie du discours artistique par rapport aux buts de propagande des commanditaires (ce qui serait une position plus humaniste que baroque). A. BEYER, après une discussion bibliographique sur le sens du maniérisme, l'explore à travers la vie de Goltzius écrite par Karl von Mander, le Vasari d'Europe du Nord, mais aussi parfait humaniste. Pour Beyer, sa vision 
de l'art n'est cependant plus celle de l'humanisme originel, car elle est suffisamment sûre d'elle pour ne plus chercher qu'en elle-même sa justification.

Le lecteur excusera ce résumé mutilant d'un recueil aux aspects très variés et dont la pluridisciplinarité est une richesse. Il permettra toutefois quelques critiques. L'assemblage des interventions manque parfois de cohérence, certaines d'entre elles n'ont que peu de rapport avec la problématique définie en introduction et il manque justement une conclusion qui dégagerait les acquis de la réflexion. À vrai dire, il faudrait peut-être une définition plus rigoureuse des concepts et une classification des objets étudiés. On peut enfin s'étonner de la part insignifiante de l'éducation et des institutions de formation dans ce panorama : car s'il est bien un secteur où l'humanisme s'est diffusé en masse, s'est survécu le plus longtemps, et où la question de la fidélité à ses origines s'est explicitement posée, c'est bien celui-là. Néanmoins, et audelà de ces lacunes, on ne peut que se féliciter de la réactivation de l'intérêt porté à l'humanisme tardif, ou tout simplement vivant au-delà de 1550 .

6 Jean-Luc LE CAM 